\title{
MAGNETOHYDRODYNAMIC EFFECTS IN RELATIVISTIC EJECTA
}

\author{
YOSUKE MIZUNO*,**, BING ZHANG ${ }^{\dagger}$, BRUNO GIACOMAZZO $^{\ddagger}$, \\ KEN-ICHI NISHIKAWA*, PHILIP E HARDEE§, \\ SHIGEHIRO NAGATAKI ${ }^{\mathbb{*}}$ and DIETER H. HARTMANN" \\ * Center for Space Plasma and Aeronomic Research, \\ University of Alabama in Huntsville, Huntsville, AL 35805, USA \\ ${ }^{\dagger}$ Department of Physics and Astronomy, University of Nevada, \\ Las Vegas, NV 89154, USA \\ ${ }^{\ddagger}$ Max-Plank-Institut für Gravitationsphysik, \\ Albert-Einstein-Institut, Potsdam-Golm, Germany \\ $\S$ Department of Physics and Astronomy, University of Alabama, \\ Tuscaloosa, AL 35487, USA \\ ฯYukawa Institute for Theoretical Physics, \\ Kyoto Univeristy, Kyoto, Japan \\ "Department of Physics and Astronomy, \\ Clemson University, Clemson, SC 29634, USA \\ **mizuno@cspar.uah.edu
}

\begin{abstract}
We study the problem of deceleration of an arbitrarily magnetized relativistic ejecta in a static unmagnetized medium and its connection to the physics of gamma-ray bursts (GRBs). By computing exact solutions of the Riemann problem describing this scenario, we find that with the same initial Lorentz factor, the reverse shock becomes progressively weaker with increasing magnetization parameter $\sigma$ (the Poynting-to-kinetic flux ratio). The reverse shock becomes a rarefaction wave when $\sigma$ exceeds a critical value defined by the balance between magnetic pressure in the ejecta and thermal pressure in the forward shock. In the rarefaction wave regime, the rarefied region is accelerated to a Lorentz factor that is significantly larger than the initial value due to the strong magnetic pressure in the ejecta. We discuss the implications for models of GRBs.
\end{abstract}

Keywords: Gamma-ray bursts; MHD; shock waves.

\section{Introduction}

Relativistic jets are believed to exist in active galactic nuclei (AGNs), black hole binaries, and gamma-ray bursts (GRBs), but their composition is still poorly understood. It has been argued that magnetic fields could play an important dynamic role in these jets, ${ }^{1-4}$ but the degree of magnetization, quantified by the magnetization parameter $\sigma$ (the ratio of electromagnetic to kinetic energy flux), is poorly

\footnotetext{
${ }^{\ddagger}$ Now at Department of Astronomy, University of Maryland, College Park, MD 20742, USA.
} 
constrained by observations. GRB afterglow modeling indicates that the ejecta are more magnetized than the ambient medium, suggesting a possibly important dynamic role for magnetic fields in GRB jets. ${ }^{5-8}$

A useful diagnostic for the degree of jet-magnetization can be obtained from the interaction between the decelerating jet and the ambient medium. Added magnetic field pressure in the jet alters the condition for the formation of a reverse shock (RS) as well as its strength. ${ }^{9}$ Analytical studies of the deceleration of a GRB fireball with arbitrary magnetization ${ }^{10}$ suggest novel behavior that does not exist in pure hydrodynamic (HD) $(\sigma=0)$ models. ${ }^{11,12}$ However, consensus on the conditions required for the existence of the RS or how Poynting flux is transferred to kinetic flux in the interaction region has not yet been achieved. ${ }^{10,13,14}$ We present a onedimensional study of the interaction between a magnetized relativistic flow and a static, unmagnetized external medium. A Riemann problem is solved analytically over a broad range of $\sigma$.

\section{The Riemann Problem}

We consider a Riemann problem consisting of two uniform initial states (left and right) with discontinuous hydrodynamic properties specified by the rest-mass density $\rho$, gas pressure $p$, specific internal energy $\epsilon$, specific enthalpy $h \equiv 1+\epsilon / c^{2}+p / \rho c^{2}$, and normal velocity $v^{N}$. The right state (the medium external to the jet) is assumed to be a cold fluid with constant density, at rest. Specifically, we select the initial conditions: $\rho_{R}=1.0 \rho_{0}, p_{R}=10^{-2} \rho_{0} c^{2}, v_{R}^{N}=v_{R}^{x}=0.0$, where $\rho_{0}$ is an arbitrary normalization constant (our simulations are scale-free) and $c$ is the speed of light. The left state (the propagating relativistic flow) is assumed to have a higher density and pressure than the right state, as well as a relativistic velocity. Specifically, $\rho_{L}=10^{2} \rho_{0}, p_{L}=1.0 \rho_{0} c^{2}$, and $v_{L}^{N}=v_{L}^{x}=0.995 c\left(\gamma_{L} \simeq 10\right)$. The fluid is described by an ideal fluid equation of state $p=(\Gamma-1) \rho \epsilon$ with $\Gamma=4 / 3$.

To investigate the effects of magnetic fields, we consider a perpendicular field component in the jet with $B^{y}=31.623,100.0,316.23$, and 447.21 in units of $\left(4 \pi \rho_{0} c^{2}\right)^{1 / 2}$ measured in the laboratory frame, corresponding to $\sigma \equiv$ $B^{2} / 4 \pi \gamma^{2} h \rho c^{2} \simeq B^{2} / 4 \pi \gamma^{2} \rho c^{2}$ being $0.1,1.0,10.0$, and 20.0, respectively. This field is motivated by the predicted toroidal field domination at the deceleration radius for GRB outflows. ${ }^{10}$ Increasing $\sigma$ increases the total (kinetic plus magnetic) energy density of the left (ejecta) state.

\section{Results}

We calculate exact solutions of this problem, using the code of Giacomazzo and Rezzolla, ${ }^{15}$ in the region $0.8 \leq x \leq 1.2$ with an initial discontinuity at $x=1.0$, where $x$ is in arbitrary units. ${ }^{16}$

\subsection{Flow-medium interaction}

The exact solutions are presented in Fig. 1. The panels display profiles of the gas density and the Lorentz factor at time $t=0.16$. Different colors represent different 


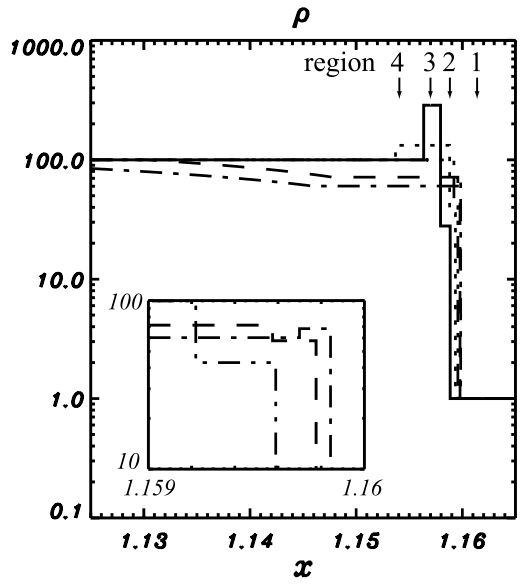

(a) $\gamma$

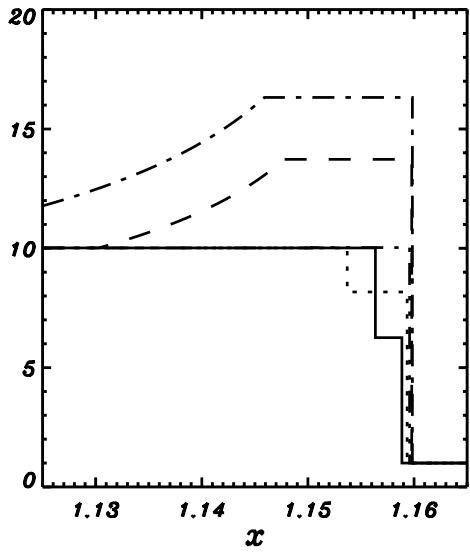

(b)

Fig. 1. Profile of (a) density and (b) Lorentz factor $(\gamma)$ of $\sigma=0.1$ (solid), 1.0 (dotted), 10.0 (dashed), and 20.0 (dash-dotted) cases at time $t=0.16$. Other parameters: $\rho_{L}=100.0, \rho_{R}=$ 1.0, $\gamma_{L}=10.0$. The critical value, $\sigma_{c} \simeq 2.7$ case is shown as dash double-dotted lines. Close-up forward shock regions are inserted. Arrows indicate four physically distinct regions: (1) unshocked medium, (2) shocked medium, (3) shocked flow and (4) unshocked flow corresponding to the $\sigma=0.1$ case.

$\sigma$ values: 0.1 (solid), 1.0 (dotted), 2.7 (dash double-dotted), 10.0 (dashed) and 20.0 (dash-dotted). The initial Lorentz factor of the left state (ejecta) is $\gamma_{L}=10$.

For $\sigma=0.1$ (solid), the solution shows a right-moving fast shock (FS: forward shock; $S_{\rightarrow}$ ), a left-moving fast shock (RS: reverse shock; $\leftarrow S$ ) relative to the contact discontinuity $(C)$. For $\sigma=1.0$ (dotted), the solution shows similar profiles $\left(\leftarrow S C S_{\rightarrow}\right)$ as $\sigma=0.1$. The FS is stronger (due to a higher jump in pressure) and slower (more deceleration relative to the frame of the contact discontinuity), while the RS is weaker but faster. These features are expected from analytical work, ${ }^{10,14}$ and agree with 1D relativistic MHD simulations. ${ }^{17,18}$ For $\sigma=10.0$ (dashed) and $\sigma=20.0$ (dash-dotted), a prominent left-going rarefaction wave $(\leftarrow R)$ is observed, instead of a left-going shock. When the rarefaction wave propagates into the ejecta, density and gas pressure decrease, and the flow velocity increases. The terminal Lorentz factor of the left (ejecta) state and the FS region reaches $\gamma \sim 14$ for $\sigma=10$ and $\gamma>16$ for $\sigma=20$. This magnetic acceleration mechanism stems from the magnetic pressure in the ejecta. ${ }^{\mathrm{a}}$

This magnetic acceleration mechanism is solely an MHD effect and requires the magnetic field to generate a rarefaction wave. This is different from the HD/MHD boost mechanism. ${ }^{20,21}$

${ }^{a}$ We note that Romero et al. ${ }^{19}$ also discovered the rarefaction wave regime discussed in this paper, but did not investigate the magnetic acceleration mechanism and its astrophysical implications in detail. 


\subsection{Conditions for reverse shock or magnetic acceleration}

In the reverse shock cases $(\sigma=0.1,1)$, the upstream magnetic pressure is lower than the gas pressure in the forward shock, while in the rarefaction wave cases $(\sigma=10,20)$, the upstream magnetic pressure exceeds the gas pressure in the FS. Thus, the balance between the upstream magnetic pressure in the unshocked flow region and the FS gas pressure in the shocked medium ${ }^{10,19}$ provides the condition separating the two regimes. This condition can be derived analytically. For the interaction between relativistic ejecta and an external medium, there exist four physically distinct regions: (1) unshocked medium, (2) shocked medium, (3) shocked ejecta, and (4) unshocked ejecta. From the relativistic shock jump conditions with $\Gamma=4 / 3$, a critical $\sigma_{c}$ value is given as $\sigma_{c}=2 \rho_{1}\left(\gamma_{4}-1\right)\left(4 \gamma_{4}+3\right) / 3 \rho_{4} \simeq 8 \gamma_{4}^{2} \rho_{1} / 3 \rho_{4}$. The condition for the existence of a reverse shock is $\sigma<\sigma_{c}$ and the condition for a rarefaction wave and magnetic acceleration is $\sigma>\sigma_{c}$. We adopted $\rho_{1}=\rho_{R}=$ 1.0, $\rho_{4}=\rho_{L}=10^{2}$, and $\gamma_{4}=\gamma_{L}=10.0$, so that the critical value is $\sigma_{c} \simeq 2.7$. Our calculations indicate that $\sigma_{c}$ marks the transition point where neither a reverse shock nor a rarefaction wave is established (dash double-dotted lines in Fig. 1).

To verify this for a larger parameter space, we investigate the $\sigma$-dependences of various quantities in detail. Figure 2(a) shows the gas pressure in the region through which the reverse shock/rarefaction wave has propagated. Initial Lorentz factors are $\gamma_{L}=5,10$, and 20, respectively. For all cases, we fix the flow density at $\rho_{L}=10^{2}$ and increase $B$ (hence $\sigma$ ). The total initial energy density of the flow increases with $\sigma$. In all cases, the gas pressure decreases with $\sigma$ smoothly without a sharp transition from the RS regime (solid lines) to the reverse rarefaction wave regime (dotted lines). The critical magnetization parameters are $\sigma_{c} \simeq 0.7,2.7,10.6$

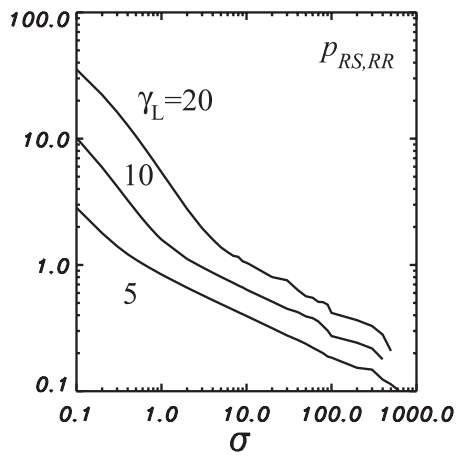

(a)

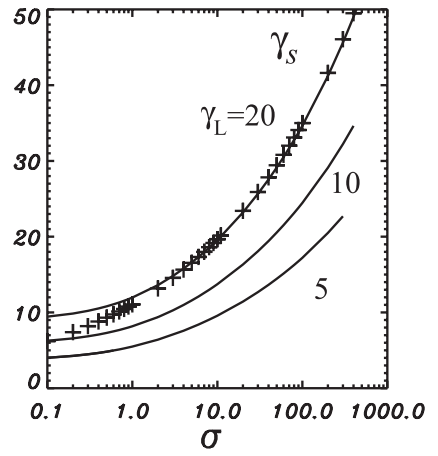

(b)

Fig. 2. The $\sigma$-dependences of (a) gas pressure in the region through which the reverse shock (RS; solid lines)/rarefaction wave ( $\mathrm{RR}$; dotted lines) has propagated and (d) the maximum Lorentz factor in the shocked region, in the exact solution. Different initial Lorentz factors have been calculated: $\gamma_{L}=5,10$, and 20. Crosses are the values of the estimated terminal Lorentz factor in the $\gamma_{L}=20$ case. A constant flow density is adopted, so that the total initial energy density of the flow increases with $\sigma$. 
for $\gamma_{L}=5,10,20$, respectively, derived from the analytical solution. We notice that in the RS regime, the strength of the shock decreases rapidly with increasing $\sigma$. The critical magnetization parameter $\sigma_{c}$ increases with $\gamma_{L}$, so that an RS can exist in the high- $\sigma$ regime if $\gamma_{L}$ is sufficiently large.

\subsection{Terminal Lorentz factor and magnetic acceleration efficiency}

To better understand the magnetic acceleration mechanism, we plot the Lorentz factor as a function of $\sigma$ in Fig. 2(b). For the magnetic acceleration case, this is the terminal Lorentz factor after acceleration. Because of the dependence of $\sigma_{c}$ on $\gamma_{L}$, a higher $\sigma$ is needed to achieve acceleration for a higher $\gamma_{L}$. The terminal Lorentz factor can be estimated analytically by requiring that the thermal pressure in the FS region balances the magnetic pressure in the region through which the rarefaction wave has propagated. For the terminal Lorentz factor $\gamma_{t}$, this condition can be expressed (roughly) as $\gamma_{t} \simeq\left(3 \gamma_{4}^{2} \sigma \rho_{4} / 8 \rho_{1}\right)^{1 / 4}$. Crosses in Fig. 2(b) denote values of estimated terminal Lorentz factors for model parameters, $\gamma_{4}=\gamma_{L}=$ $20, \rho_{1}=\rho_{R}=1.0$, and $\rho_{4}=\rho_{L}=10^{2}$, and they are in good agreement with the exact solution of the Riemann problem in the reverse rarefaction wave regime.

Regarding the acceleration efficiency, a flow with a higher initial Lorentz factor reaches a higher terminal Lorentz factor, but a lower initial Lorentz factor achieves in a higher acceleration efficiency. The acceleration efficiency is given by $\gamma_{t} / \gamma_{4} \simeq$ $\left(3 \sigma \rho_{4} / 8 \rho_{1}\right)^{1 / 4} \gamma_{4}^{-1 / 2}$ and follows from the equation for the terminal Lorentz factor.

\section{Discussion}

Our results have implications for understanding deceleration of strongly magnetized outflows, possibly present in GRBs and AGNs. Exact solutions indicate that the condition for the existence of a reverse shock is $\sigma<\sigma_{c} \cdot{ }^{10,14}$ The paucity of bright optical flashes in $\mathrm{GRBs}^{22}$ may, among other interpretions, be attributed to highly magnetized flows. If strongly magnetized jet (Poynting flux jet) is formed and leaves from the dense stellar matter like a collapsar model and goes to rarefied wind media, it is possible to form the bow-shock with the rarefaction wave on the jet head. Such bow-shock with the rarefaction wave would suppress the possibility to reradiate a significant part of the kinetic energy, which can be seen in GRBs and AGNs. For high- $\sigma$ flows, the ejecta would experience magnetic acceleration at small radii, before reaching the coasting regime, so that the coasting Lorentz factor (i.e. the "initial" Lorentz factor for the afterglow) is at least the "terminal" Lorentz factor. Here we only focus on 1D models with Cartesian geometry. We will consider the implications for GRB models in detail, when their conical jet geometry ${ }^{18}$ has been taken into account. We should note that magnetic acceleration mechanism discussed here is a simple analytical study through the Riemann problem. The problem of the magnetic acceleration of relativistic jets have been deeply discussed by many authors by using 2D RMHD simulations. ${ }^{23-28}$ 


\section{Acknowledgments}

Y. Mizuno acknowledges NASA awards NNG05GB67G, NNG05GB68G and NNX08AE57A for partial support during stay at UNLV and partial support by NSF awards AST-0506719, AST-0506666, AST-0908010, and AST-0908040, and NASA awards NNG05GK73G, NNX07AJ88G and NNX08AG83G.

\section{References}

1. R. D. Blandford and R. L. Znajek, Mon. Not. R. Astron. Soc. 179 (1977) 433.

2. R. D. Blandford and D. G. Payne, Mon. Not. R. Astron. Soc. 199 (1982) 883.

3. V. V. Usov, Nature 357 (1992) 472.

4. P. Mészáros and M. J. Rees, Astrophys. J. 482 (1997) L29.

5. Y.-Z. Fan et al., Chinese J. Astron. Astrophys. 2 (2002) 449.

6. B. Zhang, S. Kobayashi and P. Mészáros, Astrophys. J. 595 (2003) 950.

7. P. Kumar and A. Panaitescu, Mon. Not. R. Astron. Soc. 346 (2003) 905.

8. A. Gomboc et al., Astrophys. J. 687 (2008) 443.

9. C. F. Kennel and F. V. Coroniti, Astrophys. J. 283 (1984) 694.

10. B. Zhang and S. Kobayashi, Astrophys. J. 628 (2005) 315.

11. R. Sari and T. Piran, Astrophys. J. 455 (1995) L143.

12. S. Kobayashi, T. Piran and R. Sari, Astrophys. J. 513 (1999) 669.

13. M. Lyutikov, New J. Phys. 8 (2006) 119.

14. D. Giannios, P. Mimica and M. A. Aloy, Astron. Astrophys. 478 (2008) 747.

15. B. Giacomazzo and L. Rezzolla, J. Fluid Mech. 562 (2006) 223.

16. Y. Mizuno et al., Astrophys. J. 690 (2009) L47.

17. P. Mimica, M. A. Aloy and E. Müller, Astron. Astrophys. 466 (2007) 93.

18. P. Mimica, D. Giannios and M. A. Aloy, Astron. Astrophys. 494 (2009) 879.

19. R. Romero et al., J. Fluid Mech. 544 (2005) 323.

20. M. A. Aloy and L. Rezzolla, Astrophys. J. 640 (2006) L115.

21. Y. Mizuno et al., Astrophys. J. 672 (2008) 72.

22. P. W. A. Roming et al., Astrophys. J. 652 (2006) 1416.

23. S. V. Bogovalov, Astron. Astrophys. 371 (2001) 1155.

24. S. S. Komissarov et al., Mon. Not. R. Astron. Soc. 380 (2007) 51.

25. M. V. Barkov and S. S. Komissarov, Int. J. Mod. Phys. D 17 (2008) 1669.

26. S. S. Komissarov et al., Mon. Not. R. Astron. Soc. 394 (2009) 1182.

27. A. Tchekhovskoy, J. C. McKinney and R. Narayan, Mon. Not. R. Astron. Soc. 388 (2008) 551.

28. A. Tchekhovskoy, J. C. McKinney and R. Narayan, Astrophys. J. 699 (2009) 1789. 